\title{
ON RELATIVE COMPLETE REDUCIBILITY
}

\author{
CHRISTOPHER ATTENBOROUGH, MICHAEL BATE, MAIKE GRUCHOT, ALASTAIR LITTERICK, \\ GERHARD RÖHRLE
}

\begin{abstract}
Let $K$ be a reductive subgroup of a reductive group $G$ over an algebraically closed field $k$. The notion of relative complete reducibility, introduced in [4], gives a purely algebraic description of the closed $K$-orbits in $G^{n}$, where $K$ acts by simultaneous conjugation on $n$-tuples of elements from $G$. This extends work of Richardson and is also a natural generalization of Serre's notion of $G$-complete reducibility. In this paper we revisit this idea, giving a characterization of relative $G$-complete reducibility which directly generalizes equivalent formulations of $G$-complete reducibility. If the ambient group $G$ is a general linear group, this characterization yields representation-theoretic criteria. Along the way, we extend and generalize several results from [4].
\end{abstract}

\section{INTRODUCTION}

Let $G$ be a (possibly non-connected) reductive linear algebraic group and let $n \in \mathbb{N}$. The group $G$ acts by simultaneous conjugation on the $n$-fold Cartesian product $G^{n}$. In his seminal work [7, Thm. 16.4], Richardson characterized the closed $G$-orbits in $G^{n}$ in terms of the subgroup structure of $G$. In [3, Thm. 3.1] Richardson's characterization was shown to be equivalent to a notion of Serre arising from representation theory, [8], and these ideas were further extended in [4] to give a characterization of the closed $K$-orbits in $G^{n}$ for an arbitrary reductive subgroup $K$ of $G$. This gave rise to the notion of relative complete reducibility, which we briefly recall now (see Section 2 for full definitions).

Let $H$ be a subgroup of $G$ and let $K$ be a reductive subgroup of $G$. Recall that (when $G$ is connected) the parabolic subgroups of $G$ have the form $P_{\lambda}$ where $\lambda$ is a cocharacter of $G$. Following [4], we say that $H$ is relatively $G$-completely reducible with respect to $K$ if for every cocharacter $\lambda$ of $K$ such that $H$ is contained in the subgroup $P_{\lambda}$ of $G$, there exists a cocharacter $\mu$ of $K$ such that $P_{\lambda}=P_{\mu}$ and $H \subseteq L_{\mu}$, a Levi subgroup of $P_{\lambda}$. For $K=G$, this definition coincides with the usual notion of $G$-complete reducibility due to Serre, cf. [3], [8].

The following algebraic characterization of the closed $K$-orbits in $G^{n}$ in terms of relative $G$-complete reducibility was given in [4, Thm. 1.1]:

Theorem 1.1. Let $K$ be a reductive subgroup of $G$. Let $H$ be the algebraic subgroup of $G$ generated by elements $x_{1}, \ldots, x_{n} \in G$. Then $K \cdot\left(x_{1}, \ldots, x_{n}\right)$ is closed in $G^{n}$ if and only if $H$ is relatively $G$-completely reducible with respect to $K$.

2010 Mathematics Subject Classification. 20G15 (14L24).

Key words and phrases. reductive algebraic groups, $G$-complete reducibility, closed $G$-orbits.

The first author is supported by an EPSRC Doctoral Training award. The fourth author is supported by the Alexander von Humboldt Foundation. 
Recall that in the particular case $K=G$ and $G=\mathrm{GL}(V)$, a subgroup $H$ of $G$ is $G$ completely reducible if and only if $V$ is a completely reducible $H$-module. This latter property can be defined equivalently by either (i) every $H$-stable flag of subspaces of $V$ admits an $H$-stable complement; (ii) every $H$-stable subspace of $V$ admits an $H$-stable complement; or (iii) $V$ is a direct sum of irreducible $H$-submodules. Our main result, Theorem 1.2 below, generalizes these equivalences firstly to other connected reductive algebraic groups $G$, and also to the relative setting. When $G=\mathrm{GL}(V)$ this characterizes relative $\mathrm{GL}(V)$-complete reducibility (with respect to $K$ ) in terms of certain flags of submodules of $V$.

In order to state our results, we need some notation. Recall that an R-parabolic subgroup of $G$ is a subgroup of the form $P_{\lambda}$ for a cocharacter $\lambda$ of $G$ (see Section 2). Let $\mathcal{P}$ be the poset of R-parabolic subgroups of $G$ under inclusion. For a reductive subgroup $K$ of $G$, write $\mathcal{P}_{K}$ for the set of R-parabolic subgroups $P_{\lambda}$ with $\lambda \in Y(K)$. Then $\mathcal{P}_{K}$ is also a poset under inclusion; write $\mathcal{M}_{K}$ for its maximal elements. If $K=G$ is connected then $\mathcal{P}_{K}$ is the poset dual to the spherical building of $G$, and $\mathcal{M}_{K}$ is the set of maximal (proper) parabolic subgroups of $G$; in this case all members of $\mathcal{M}_{K}$ have the same rank (i.e. height in the poset $\mathcal{P})$. This need not hold in general, cf. Example 4.3.

Conjugation gives a natural action of $G$ and its subgroups on $\mathcal{P}$. Recall that two Rparabolic subgroups are called opposite if their intersection is an R-Levi subgroup of $G$. It follows easily from the definitions that a subgroup $H$ of $G$ is relatively $G$-completely reducible with respect to $K$ precisely when each member of $\mathcal{P}_{K}$ containing $H$ has an opposite in $\mathcal{P}_{K}$ containing $H$. The following is our main result.

Theorem 1.2. Let $K \leq G$ be reductive algebraic groups with $G$ connected, and let $H$ be a subgroup of $G$. Then the following are equivalent:

(i) $H$ is relatively $G$-completely reducible with respect to $K$.

(ii) Each member of $\mathcal{M}_{K}$ containing $H$ has an opposite in $\mathcal{M}_{K}$ containing $H$.

(iii) There is an $R$-Levi subgroup $L_{\mu}$ with $\mu \in Y(K)$, such that $H \leq L_{\mu}$ and $H$ is relatively $L_{\mu}$-irreducible with respect to $K \cap L_{\mu}$.

Remarks 1.3.

(i) Conditions (i)-(iii) specialize to the representation-theoretic notions discussed above if we take $K=G=\mathrm{GL}(V)$.

(ii) A result of Serre [8, Thm. 2.2] states that when $K=G$ is a connected reductive group, a subgroup is $G$-completely reducible precisely when it lies in a Levi subgroup of each maximal parabolic subgroup containing it. This result is central to the study of complete reducibility via the spherical building of $G$, as outlined in loc. cit. The equivalence of (i) and (ii) in Theorem 1.2 can be recast as a relative analogue of this building-theoretical result, generalizing Serre's result.

(iii) In the case $G=\mathrm{GL}(V)$, Theorem 1.2 provides very concrete criteria for relative complete reducibility. The general form of these criteria is given in Corollary 1.5 below, and is illustrated with explicit examples in Corollary 1.7 (when $K=\mathrm{SO}(V)$ or $\operatorname{Sp}(V)$ ) and in Appendix A (when $K$ is simple of type $G_{2}$ ).

The proof of Theorem 1.2 makes use of the fact that $G$ is connected. However, the implications (i) $\Leftrightarrow$ (iii) $\Rightarrow$ (ii) all hold without this assumption. For the missing implication, 
essentially the only issue is that in a non-connected group, not every R-parabolic subgroup in $\mathcal{P}_{K}$ need arise as the intersection of members of $\mathcal{M}_{K}$ (see Example 2.3). However, this does hold when $G$ is connected (Lemma 2.2). Theorem 1.2 is therefore a consequence of our next result. For this, define the set

$$
\mathcal{P}_{K}^{\prime}:=\left\{P \in \mathcal{P}_{K} \mid P=\bigcap\left\{Q \in \mathcal{M}_{K} \mid P \leq Q\right\}\right\} .
$$

Theorem 1.4. Let $K \leq G$ be reductive algebraic groups, and let $H$ be a subgroup of $G$. Then each member of $\mathcal{M}_{K}$ containing $H$ has an opposite in $\mathcal{M}_{K}$ containing $H$ if and only if each member of $\mathcal{P}_{K}^{\prime}$ containing $H$ has an opposite in $\mathcal{P}_{K}^{\prime}$ containing $H$.

As a corollary, the conclusion of Theorem 1.2 also holds for (possibly non-connected) reductive groups $G$ such that $\mathcal{P}_{K}=\mathcal{P}_{K}^{\prime}$.

In the particular case $G=\mathrm{GL}(V)$, Theorem 1.2 gives a representation-theoretic characterization of relative complete reducibility, as follows. Recall that a parabolic subgroup of $\mathrm{GL}(V)$ is the stabilizer of a flag of subspaces in $V$. The poset of flags in $V$ is the dual of the poset of parabolic subgroups in GL(V), i.e., we set $f \preceq f^{\prime}$ provided $\operatorname{Stab}_{G}(f) \supseteq \operatorname{Stab}_{G}\left(f^{\prime}\right)$. For $K$ a reductive subgroup of GL $(V)$, we denote by $\mathcal{F}_{K}$ the set of flags in $V$ which stem from $K$, i.e., which correspond to parabolic subgroups $P_{\lambda}$ for $\lambda$ a cocharacter of $K$. A flag $f$ in $\mathcal{F}_{K}$ is called minimal in $\mathcal{F}_{K}$ provided $f^{\prime} \preceq f$ for $f^{\prime}$ in $\mathcal{F}_{K}$ implies $f^{\prime}=f$. Let $\mathcal{M} \mathcal{F}_{K} \subseteq \mathcal{F}_{K}$ be the set of minimal flags in $\mathcal{F}_{K}$. Again, Example 4.3 shows that members of $\mathcal{M F}_{K}$ may have varying lengths. Of course, $\mathcal{M F}_{\mathrm{GL}(V)}$ is the set of flags of length 1 in $V$, corresponding to the set of maximal parabolic subgroups in $\mathrm{GL}(V)$.

Corollary 1.5. Let $H$ and $K$ be closed subgroups of $\mathrm{GL}(V)$ with $K$ reductive. Then the following are equivalent:

(i) $H$ is relatively $\mathrm{GL}(V)$-completely reducible with respect to $K$.

(ii) Every $H$-stable flag in $\mathcal{M F}_{K}$ has an $H$-stable opposite in $\mathcal{M F}_{K}$.

(iii) There is a maximal torus $S$ of $C_{K}(H)$ such that $H$ preserves the direct-sum decomposition of $V$ into simultaneous $S$-eigenspaces, and this decomposition gives a flag which is maximal among $H$-stable flags in $\mathcal{F}_{K}$.

When $K=G=\mathrm{GL}(V)$, the possibilities for the torus $S$ in (iii) are products of the centres of the GL( $\left.V_{i}\right)$ corresponding to a direct-sum decomposition of $V$ into irreducible modules $V=V_{1} \oplus \ldots \oplus V_{r}(r \geq 1)$; this gives the usual representation-theoretic characterization.

The next result more closely mirrors the representation-theoretic statement that $V$ is a completely reducible $H$-module if and only if every $H$-submodule has a complement. For this, however, we require an additional hypothesis. Assume once more that $G=\operatorname{GL}(V)$, and write $\mathcal{S}_{K}$ for the set of subspaces of $V$ which arise in flags from $\mathcal{F}_{K}$.

Corollary 1.6. Let $H, K$ be subgroups of $\mathrm{GL}(V)$ with $K$ reductive. Suppose $\mathcal{M F}_{K} \subseteq$ $\mathcal{M F}_{\mathrm{GL}(V)}$. Then the following are equivalent:

(i) $H$ is relatively $\mathrm{GL}(V)$-completely reducible with respect to $K$.

(ii) For each $U \in \mathcal{S}_{K}$ which is stabilized by $H$ there exists $W \in \mathcal{S}_{K}$ such that $H$ stabilizes $W$ and $V=U \oplus W$, as an $H$-module. 
Corollary 1.6 readily follows from Corollary 1.5 and Corollary 4.2. Note that the implication (i) $\Rightarrow$ (ii) can fail without the hypothesis on $\mathcal{M F}_{K}$, see Example 4.3. The essential problem is that an $H$-stable member of $\mathcal{S}_{K}$ need not arise from an $H$-stable flag in $\mathcal{F}_{K}$.

Corollary 1.6 may be viewed as a generalization of [4, Prop. 5.1]. The latter gives a representation-theoretic characterization of relative $\mathrm{GL}(V)$-complete reducibility in the case that $K=\mathrm{GL}(U)$ for a subspace $U$ of $V$, which is closely related to the condition in Corollary 1.6, see Lemma 4.5 and Corollary 4.2 .

Particularly natural candidates for the subgroup $K$ in $\mathrm{GL}(V)$ are the classical groups $\mathrm{SO}(V)$ and $\operatorname{Sp}(V)$. This is the theme of our next result, which gives a characterization of relative $\mathrm{GL}(V)$-complete reducibility with respect to $\mathrm{SO}(V)$ or $\mathrm{Sp}(V)$ in terms of totally singular or totally isotropic subspaces.

Corollary 1.7. Let $H$ be a subgroup of $\mathrm{GL}(V)$ and let $K=\mathrm{Sp}(V)$ (resp. $\mathrm{SO}(V)$ ). Then the following are equivalent:

(i) $H$ is relatively $\mathrm{GL}(V)$-completely reducible with respect to $K$.

(ii) Whenever $H$ stabilizes a totally isotropic (resp. totally singular) subspace $U$ and its annihilator $U^{\perp}$, there exists a totally isotropic (resp. totally singular) subspace $W \subseteq V$ such that $H$ stabilizes $W$ and $W^{\perp}$, and $V=W \oplus U^{\perp}=U \oplus W^{\perp}$ as $H$-modules.

In the setting of Corollary 1.7, flags in $\mathcal{F}_{K}$ have the form

$$
U_{1} \subseteq \ldots \subseteq U_{r} \subseteq U_{r}^{\perp} \subseteq \ldots \subseteq U_{1}^{\perp} \subseteq V
$$

so that minimal flags in $\mathcal{F}_{K}$ are of the form $U \subseteq U^{\perp} \subseteq V$ for $U$ a totally isotropic (resp. totally singular) subspace, hence the result is immediate from Corollary 1.5.

Note that we cannot relax the requirement in Corollary 1.7(ii) that $H$ stabilizes $U^{\perp}$ and $W^{\perp}$, since $H$ does not need to leave the form on $V$ invariant (i.e. $H$ need not be a subgroup of $K)$.

In Section 5, we present a brief investigation of the notion of relative $G$-complete reducibility over an arbitrary field, obtaining rational versions of Theorems 1.2 and 1.4. In Appendix A we give an illustrative example of Theorem 1.2, with $K$ simple of exceptional type and $G=\mathrm{GL}(V)$ for a $K$-module $V$.

\section{PRELiminaries}

We work over a field $k$, which is taken to be algebraically closed except in Section 5 . Let $G$ be a reductive algebraic group defined over $k$ - we allow the possibility that $G$ is not connected. Let $H$ be a closed subgroup of $G$. We write $H^{\circ}$ for the identity component of $H$.

For the set of cocharacters (one-parameter subgroups) of $G$ we write $Y(G)$.

Suppose $G$ acts on a variety $X$ and let $x \in X$. Then for each cocharacter $\lambda \in Y(G)$ we define a morphism of varieties $\phi_{x, \lambda}: k^{*} \rightarrow X$ via $\phi_{x, \lambda}(a)=\lambda(a) \cdot x$. If this morphism extends to a morphism $\bar{\phi}_{x, \lambda}: k \rightarrow X$, then we say that the $\operatorname{limit}_{a \rightarrow 0} \lambda(a) \cdot x$ exists and set this limit equal $\bar{\phi}_{x, \lambda}(0)$. For each cocharacter $\lambda \in Y(G)$, let $P_{\lambda}=\left\{g \in G \mid \lim _{a \rightarrow 0} \lambda(a) g \lambda(a)^{-1}\right.$ exists $\}$ and $L_{\lambda}=\left\{g \in G \mid \lim _{a \rightarrow 0} \lambda(a) g \lambda(a)^{-1}=g\right\}$. Following [3, §6], we call $P_{\lambda}$ an $R$-parabolic 
subgroup of $G$ and $L_{\lambda}$ an $R$-Levi subgroup of $G$. As mentioned previously, if $G$ is connected then these R-parabolic subgroups and their R-Levi subgroups are precisely the parabolic subgroups and their Levi subgroups. If $K$ is a reductive subgroup of $G$ and $\lambda \in Y(K)$, we always denote by $P_{\lambda}$ the R-parabolic subgroup of $G$ attached to $\lambda$; if we need to consider the corresponding R-parabolic subgroup of $K$ we write $P_{\lambda}(K)$ (and similarly for R-Levi subgroups). We recall the following central notions from [4].

Definition 2.1. Let $H$ and $K$ be subgroups of $G$ with $K$ reductive. We say that $H$ is relatively $G$-completely reducible with respect to $K$ if, for every $\lambda \in Y(K)$ such that $H$ is contained in $P_{\lambda}$, there exists $\mu \in Y(K)$ such that $P_{\lambda}=P_{\mu}$ and $H$ is contained in $L_{\mu}$. We sometimes use the abbreviations relatively $G$-cr with respect to $K$. We say that $H$ is relatively $G$-irreducible with respect to $K$ if $H$ is not contained in any subgroup $P_{\lambda}$ with $\lambda \in Y(K)$.

Note that $H$ is relatively $G$-completely reducible with respect to $K$ if and only if $H$ is relatively $G$-completely reducible with respect to $K^{\circ}$, and similarly for relative $G$-irreducibility. In the case when $K=G$, Definition 2.1 coincides with the usual definitions, cf. [3].

Lemma 2.2. Let $K \leq G$ be reductive groups with $G$ connected, and let $\mathcal{P}_{K}, \mathcal{M}_{K}$ and $\mathcal{P}_{K}^{\prime}$ be as in the introduction.

(i) Let $P \in \mathcal{P}_{K}$ and let $Q$ be a maximal $R$-parabolic subgroup of $G$ containing $P$. Then there exists $P^{\prime} \in \mathcal{M}_{K}$ such that $P \leq P^{\prime} \leq Q$.

(ii) Let $P_{\lambda} \in \mathcal{P}_{K}$ with $\lambda\left(k^{*}\right) \leq S$, a fixed maximal torus of $K$. Then $P=\bigcap_{i=1}^{m} P_{\lambda_{i}}$ for some $\lambda_{i} \in Y(S)$ such that $P_{\lambda_{i}} \in \mathcal{M}_{K}$ for all $i$. In particular, $\mathcal{P}_{K}=\mathcal{P}_{K}^{\prime}$.

Proof. (i) Note that maximal chains of R-parabolic subgroups in the posets $\mathcal{P}$ and $\mathcal{P}_{K}$ are finite; we argue by induction on the height of $P$ in $\mathcal{P}_{K}$. If $P \in \mathcal{M}_{K}$ then there is nothing to prove. Now suppose that $P$ is not maximal in $\mathcal{P}_{K}$. Then there exist $\lambda, \nu \in Y(K)$ such that $P=P_{\lambda} \leq P_{\nu}$ and $P_{\nu} \in \mathcal{M}_{K}$. If $P_{\nu} \leq Q$ then we are done. So we assume that $P_{\nu} \not \mathbf{Q}$.

Let $\mu \in Y(G)$ such that $Q=P_{\mu}$. There is a maximal torus $T$ of $G$ in $P_{\lambda}$ such that $T \cap K$ is a maximal torus of $K$ and such that $T \leq P_{\lambda} \leq P_{\nu}$. Let $T \leq B \leq P_{\lambda}$ be a Borel subgroup of $G$.

Let $\Phi$ be the set of roots of $G$ with respect to $T$ and let $\Phi^{+}$be the set of positive roots of $G$ with respect to $B$, with corresponding set of simple roots $\Delta$. For $I \subseteq \Delta$, we denote by $P_{I}$ the standard parabolic subgroup of $G$ generated by $T$ and the root groups corresponding to roots in $-I \cup \Phi^{+}$. By the proof of [9, Prop. 8.4.5], setting $I_{\xi}:=\{\alpha \in \Delta \mid\langle\alpha, \xi\rangle=0\}$ for $\xi \in Y(T)$, where $\langle\alpha, \xi\rangle$ denotes the standard pairing between characters and cocharacters of $T$, if $P_{\xi}$ contains $B$ then $P_{I_{\xi}}=P_{\xi}$. Because of this, we have $I_{\lambda} \subseteq I_{\nu}$ and $I_{\lambda} \subsetneq I_{\mu}$. Note also that since $P_{\mu}$ is a maximal parabolic, we have $\Delta \backslash I_{\mu}=\left\{\alpha_{0}\right\}$, a single simple root. Since $P_{\nu}$ is not contained in $P_{\mu}$, we have $\alpha_{0} \in I_{\nu}$. Hence $\Delta=I_{\mu} \cup I_{\nu}$.

Given any $\alpha \in I_{\mu} \backslash I_{\lambda}$, we have $\langle\alpha, \lambda\rangle>0$. Further, since $I_{\nu}$ is not contained in $I_{\mu}$, there is at least one choice of $\alpha \in I_{\mu} \backslash I_{\lambda}$ with $\langle\alpha, \nu\rangle>0$ as well. Let $n_{1}, n_{2} \in \mathbb{N}$ be such that $\frac{n_{1}}{n_{2}} \in \mathbb{Q}_{>0}$ is the maximum of all ratios $\frac{\langle\alpha, \nu\rangle}{\langle\alpha, \lambda\rangle}$ over all $\alpha \in I_{\mu} \backslash I_{\lambda}$, and set $\chi:=n_{1} \lambda-n_{2} \nu$. Note that $\chi \in Y(K)$ and thus $P_{\chi} \in \mathcal{P}_{K}$. We show in the following paragraph that $P_{\chi}$ is a standard parabolic subgroup of $G$ properly containing $P_{\lambda}$ and contained in $P_{\mu}$. This is enough to complete the proof, by induction. 
To see that $P_{\chi}$ is standard, let $\beta \in \Delta$. Then $\beta \in I_{\mu}$ or $\beta \in I_{\nu} \backslash I_{\mu}$, because $\Delta=I_{\mu} \cup I_{\nu}$, as observed above. In the first case, we have $\langle\beta, \chi\rangle \geq 0$, by the choice of $\frac{n_{1}}{n_{2}}$ above. In the second case, $\langle\beta, \nu\rangle=0$, so we have $\langle\beta, \chi\rangle=n_{1}\langle\beta, \lambda\rangle \geq 0$. Hence $\langle\beta, \chi\rangle \geq 0$ for all $\beta \in \Delta$, which shows that $P_{\chi}$ is standard. Now, to see that $P_{\lambda}$ is properly contained in $P_{\chi}$ it suffices to check that $I_{\lambda}$ is properly contained in $I_{\chi}$. The containment is clear because $I_{\lambda} \subseteq I_{\nu}$ and $\chi$ is a combination of $\lambda$ and $\nu$. Moreover, if we let $\alpha \in I_{\mu} \backslash I_{\lambda}$ be such that the maximum value $\frac{n_{1}}{n_{2}}$ above is attained, then $\langle\alpha, \lambda\rangle>0$, but $\langle\alpha, \chi\rangle=0$, so the containment is proper. Finally, to see that $P_{\chi} \subseteq P_{\mu}$, we only need to see that $\left\langle\chi, \alpha_{0}\right\rangle>0$, where $\alpha_{0} \in \Delta$ is the unique simple root outside $I_{\mu}$, as above. But we have argued that $\alpha_{0} \in I_{\nu}$, and $\alpha_{0} \notin I_{\lambda}$ since $P_{\lambda}$ is proper in $P_{\mu}$. Hence $\left\langle\alpha_{0}, \chi\right\rangle=n_{1}\left\langle\alpha_{0}, \lambda\right\rangle>0$, and we are done.

Thus, by induction there exists $P^{\prime} \in \mathcal{M}_{K}$ such that $P_{\chi} \leq P^{\prime} \leq P_{\mu}=Q$, which completes the proof of (i).

(ii) Since $G$ is connected, the R-parabolic subgroup $P_{\lambda}$ is equal to the intersection of all maximal R-parabolic subgroups of $G$ which contain it. By (i), for each such maximal Rparabolic subgroup $Q_{i}(i=1, \ldots, m)$ we can find an R-parabolic subgroup $P_{i} \in \mathcal{M}_{K}$ with $P_{\lambda} \leq P_{i} \leq Q_{i}$. Then $P_{\lambda}=\bigcap_{i=1}^{m} P_{i}$. Each subgroup $P_{i}$ has the form $P_{\lambda_{i}}$ for some cocharacter $\lambda_{i}$ of $K$. Now $S \leq P_{\lambda} \leq P_{\lambda_{i}}$ for each $i$, so $S$ is a maximal torus of $P_{\lambda_{i}}(K)$. Since $\lambda_{i}\left(k^{*}\right)$ is a sub-torus of $P_{\lambda_{i}}(K)$, it is $P_{\lambda_{i}}(K)$-conjugate to a subgroup of $S$. So we can replace $\lambda_{i}$ by an appropriate $P_{\lambda_{i}}$-conjugate so that $\lambda_{i} \in Y(S)$ for each $i$, as required.

The following example shows that Lemma 2.2(ii) can fail when $G$ is not connected.

Example 2.3. Let $T$ be a 1-dimensional torus, let $\langle x\rangle$ be cyclic of order 8 and let $G=K=$ $T^{8} \rtimes\langle x\rangle$, with $x$ permuting the factors of $T^{8}$ in the obvious manner. Then each R-parabolic subgroup of $G$ contains $T^{8}=G^{\circ}$, and every subgroup $G^{\circ} \leq P \leq G$ arises as an R-parabolic subgroup $P_{\lambda}$, depending on whether the 1-dimensional torus $\lambda\left(k^{*}\right)$ is centralized by $x, x^{2}, x^{4}$ or none of these. Then $T^{8} \rtimes\left\langle x^{2}\right\rangle$ is the unique maximal proper R-parabolic subgroup of $G$, and in particular its subgroup $T^{8} \rtimes\left\langle x^{4}\right\rangle$ is not the intersection of the maximal R-parabolic subgroups of $G$ containing it.

Lemma 2.4. Let $G$ be reductive, and let $\lambda_{i}(i=1, \ldots, m)$ be pairwise commuting cocharacters of $G$ such that there exists a Borel subgroup $B$ of $G$ with $B \subseteq P_{\lambda_{i}}$ for each $i$. Then there exist postive integers $n_{i}(i=1, \ldots, m)$ such that $P_{\lambda}=\bigcap_{i=1}^{m} P_{\lambda_{i}}$, where $\lambda=\sum_{i=1}^{m} n_{i} \lambda_{i}$.

Proof. If $G$ is connected, this follows quickly from the well-understood theory of standard parabolic subgroups (parabolic subgroups containing a fixed Borel subgroup); the subgroups $P_{\lambda_{i}}$ correspond to choosing subsets of simple roots of $G$, and $P_{\lambda}$ corresponds to choosing the union of these sets (independently of the choice of positive integers $n_{i}$ ). For general $G$, by [3, Lem. 6.2(iii)] it suffices to show that $R_{u}\left(P_{\lambda}\right)=R_{u}\left(\bigcap_{i=1}^{m} P_{\lambda_{i}}\right)$ and $L_{\lambda}=\left(\bigcap_{i=1}^{m} L_{\lambda_{i}}\right)$ for some choice of integers $n_{i}$. Moreover, it suffices to treat the case $m=2$, and the general case then follows by an easy induction. Now, since $R_{u}(P)=R_{u}\left(P^{\circ}\right)=R_{u}\left(P \cap G^{\circ}\right)$ for every Rparabolic subgroup $P$, the equality $R_{u}\left(P_{\lambda}\right)=R_{u}\left(P_{\lambda_{1}} \cap P_{\lambda_{2}}\right)$ follows from the corresponding result for connected $G$ and [3, Lem. 6.2(iii)]. Finally [3, Lem. 6.2(i)] tells us that the equality $L_{\lambda}=L_{\lambda_{1}} \cap L_{\lambda_{2}}$ holds for sufficiently large $n_{1}$. 
The following example shows that the positive integers $n_{i}$ cannot be chosen arbitrarily when $G$ is not connected. We thank Dr. Tomohiro Uchiyama for pointing this out.

Example 2.5. Let $G=\mathrm{SL}_{3}(k)\langle\sigma\rangle$, where $\sigma$ is the inverse-transpose automorphism composed with conjugation by $\left(\begin{array}{lll}0 & 0 & 1 \\ 0 & 1 & 0 \\ 1 & 0 & 0\end{array}\right)$. Then $\sigma$ normalises the diagonal maximal torus $T$ and the upper triangular Borel subgroup $B$, swapping the root groups $U_{\alpha}$ and $U_{\beta}$ corresponding to the $(1,2)$ and $(2,3)$ matrix coordinates. Let $\lambda(c)=\operatorname{diag}\left(c, c, c^{-2}\right), \mu(c)=\operatorname{diag}\left(c^{2}, c^{-1}, c^{-1}\right)$ for $c \in k^{*}$, so that $\lambda, \mu \in Y(T)$ with $P_{\lambda}^{\circ}=\left\langle T, U_{ \pm \alpha}, U_{\beta}\right\rangle$ and $P_{\mu}^{\circ}=\left\langle T, U_{ \pm \beta}, U_{\alpha}\right\rangle$. It is evident that $\sigma$ does not normalise $P_{\lambda}^{\circ}$ or $P_{\mu}^{\circ}$, hence is not contained in $P_{\lambda}$ or in $P_{\mu}$. On the other hand, it is easily checked that $\sigma$ centralises $(\lambda+\mu)\left(k^{*}\right)$; in particular $P_{\lambda+\mu}$ contains $\sigma$ hence is strictly larger than $P_{\lambda} \cap P_{\mu}$. On the other hand, for any choice of distinct positive integers $n_{1}, n_{2}$, it is the case that $P_{n_{1} \lambda+n_{2} \mu}=P_{\lambda} \cap P_{\mu}=B$.

We require the following useful result, which is [4, Lem. 3.3].

Lemma 2.6. Let $K \leq G$ be reductive groups.

(i) Let $\lambda, \mu \in Y(K)$ such that $P_{\lambda}=P_{\mu}$ and $u \in R_{u}\left(P_{\lambda}(K)\right)$ such that $u L_{\lambda}(K) u^{-1}=$ $L_{\mu}(K)$. Then $u L_{\lambda} u^{-1}=L_{\mu}$.

(ii) Let $H$ be a subgroup of $G$. Then $H$ is relatively $G$-completely reducible with respect to $K$ if and only if for every $\lambda \in Y(K)$ such that $H \subseteq P_{\lambda}$ there exists $u \in R_{u}\left(P_{\lambda}(K)\right)$ such that $H \subseteq L_{u \cdot \lambda}$.

The following is the final ingredient needed in the proof of Theorems 1.2 and 1.4.

Lemma 2.7. Let $K \leq G$ be reductive groups. If $P \in \mathcal{M}_{K}$ (resp. $P \in \mathcal{P}_{K}^{\prime}$ ) and $Q \in \mathcal{P}_{K}$ is opposite to $P$, then $Q \in \mathcal{M}_{K}$ (resp. $Q \in \mathcal{P}_{K}^{\prime}$ ).

Proof. We prove firstly that if $P \in \mathcal{M}_{K}$, and if $Q \in \mathcal{P}_{K}$ is opposite to $P$, then $Q \in \mathcal{M}_{K}$. Suppose not. Then there exists $Q^{\prime} \in \mathcal{M}_{K}$ such that $Q \lessgtr Q^{\prime}$. Let $T$ be a maximal torus of $K$ contained in $Q$ and $Q^{\prime}$. Then there exits $\lambda \in Y(T)$ such that $P=P_{\lambda}$ and $Q=P_{-\lambda}$. Since $T \leq P_{-\lambda}<Q^{\prime}$, there exists $\mu \in Y(T)$ such that $Q^{\prime}=P_{\mu}$. Let $P^{\prime}=P_{-\mu} \in \mathcal{P}_{K}$. Then $H \leq P_{\lambda}<P_{-\mu}$, contradicting $P \in \mathcal{M}_{K}$.

Now suppose $P \in \mathcal{P}_{K}^{\prime}$. Since $G$ is noetherian, we can write $P$ as a finite intersection $P=\bigcap_{i=1}^{m} P_{i}$ with $P_{i} \in \mathcal{M}_{K}$ for each $i$. Let $Q \in \mathcal{P}_{K}$ be opposite to $P$, and let $T$ be a maximal torus of $K$ contained in $P \cap Q$. Then for each $i$ we have $P_{i}=P_{\lambda_{i}}$ for some $\lambda_{i} \in Y(T)$, and by Lemma 2.4 we have $P=P_{\lambda}$ where $\lambda=\sum_{i=1}^{m} n_{i} \lambda_{i}$ for some positive integers $n_{i}$. By Lemma 2.6 and the uniqueness of opposite R-parabolic subgroups containing a given R-Levi subgroup [3, Lem. 6.11], we can conjugate $\lambda$ (and each $\lambda_{i}$ ) by some fixed element of $R_{u}\left(P_{\lambda}(K)\right)$ such that $Q=P_{-\lambda}=\bigcap_{i=1}^{m} P_{-\lambda_{i}}$. By the paragraph above, each subgroup $P_{-\lambda_{i}} \in \mathcal{M}_{K}$ for each $i$, hence $Q \in \mathcal{P}_{K}^{\prime}$.

\section{Proof of Theorems 1.2 And 1.4}

We now prove Theorems 1.2 and 1.4. We begin with the proof of Theorem 1.4, from which Theorem 1.2 follows in short order. 
Recall that $G$ is a (not necessarily connected) reductive algebraic group and $H$ and $K$ are subgroups of $G$, with $K$ also reductive (but not necessarily connected). Also $\mathcal{P}_{K}$ is the set of R-parabolic subgroups of $G$ of the form $P_{\lambda}$ with $\lambda \in Y(K), \mathcal{M}_{K}$ is the set of maximal members of $\mathcal{P}_{K}$ under inclusion, and $\mathcal{P}_{K}^{\prime}$ is the set of members of $\mathcal{P}_{K}$ which can be realized as intersections of members of $\mathcal{M}_{K}$.

Proof of Theorem 1.4. Suppose that each member of $\mathcal{P}_{K}^{\prime}$ which contains $H$ admits an opposite in $\mathcal{P}_{K}^{\prime}$ containing $H$. Since $\mathcal{M}_{K} \subseteq \mathcal{P}_{K}^{\prime}$ by definition, if $P \in \mathcal{M}_{K}$ and $P \geq H$ then $P \in \mathcal{P}_{K}^{\prime}$, hence admits an opposite $Q \in \mathcal{P}_{K}^{\prime}$ which contains $H$. By Lemma 2.7 we have $Q \in \mathcal{M}_{K}$, as required.

Conversely, suppose that each member of $\mathcal{M}_{K}$ containing $H$ admits an opposite in $\mathcal{M}_{K}$ which contains $H$, and let $P \in \mathcal{P}_{K}^{\prime}$ such that $P \geq H$. By definition of $\mathcal{P}_{K}^{\prime}$ we can write $P=\bigcap_{i=1}^{m} P_{i}$ for some $m \in \mathbb{N}$ and $P_{i} \in \mathcal{M}_{K}$ for each $i$. Taking $m$ to be minimal, we proceed by induction on $m$, the case $m=1$ being our starting hypothesis.

By Lemma 2.6 we can fix a maximal torus $T$ of $K$ contained in $P$ such that $P=P_{\lambda}$ and $P_{i}=P_{\lambda_{i}}$ for some cocharacters $\lambda$ and $\lambda_{i} \in Y(T)$ for each $i$. Write this intersection as $P_{\lambda}=$ $P_{\lambda_{1}} \cap \bigcap_{i=2}^{m} P_{\lambda_{i}}$. By Lemma 2.4 we are free to write $\bigcap_{i=2}^{m} P_{\lambda_{i}}=P_{\nu}$ where $\nu=n_{2} \lambda_{2}+\ldots+n_{m} \lambda_{m}$ for some positive integers $n_{i}$, and we are also free to replace $\lambda$ (without changing $P_{\lambda}$ ) such that $\lambda=n_{1} \lambda_{1}+n_{\nu} \nu$ for some positive integers $n_{1}, n_{\nu}$.

Since $P_{\lambda_{1}} \in \mathcal{M}_{K}$, by hypothesis $H$ lies in some R-Levi subgroup of $P_{\lambda_{1}}$. Since $P_{\lambda} \leq P_{\lambda_{1}}$ we have $R_{u}\left(P_{\lambda_{1}}(K)\right) \leq R_{u}\left(P_{\lambda}(K)\right)$. Thus we are free to replace $H$ by an $R_{u}\left(P_{\lambda_{1}}(K)\right)$-conjugate so that $H \leq L_{\lambda_{1}}$, and this does not change whether $H$ lies in an R-Levi subgroup of $P_{\lambda}$ corresponding to a cocharacter of $K$. Moreover, replacing $H$ with such a conjugate, we still have $H \leq P_{\lambda} \leq P_{\nu}$. Now since $H \leq L_{\lambda_{1}}$, we have $\lambda_{1} \in Y\left(C_{K}(H) \cap L_{\nu}(K)\right)=Y\left(C_{L_{\nu}(K)}(H)\right)$.

Now, we can apply the induction hypothesis to $P_{\nu}$, so that there exists $\sigma \in Y(K)$ such that $P_{\sigma} \in \mathcal{P}_{K}^{\prime}$ is opposite to $P_{\nu}$ and $H \leq L_{\sigma}$. Then there exists $u \in R_{u}\left(P_{\nu}(K)\right)$ such that $u \cdot \nu \in Y\left(L_{\sigma}(K)\right)$. So we set $\tau=-(u \cdot \nu)$ as cocharacters of some maximal torus of $L_{\sigma}(K)$. By the uniqueness of opposite parabolic subgroups containing a fixed maximal torus [3, Lem. 6.11] we have $P_{\tau}=P_{\sigma}, L_{\tau}=L_{\sigma}$. Since $\tau, \lambda_{1} \in Y\left(C_{P_{\nu}(K)}(H)\right), \tau$ is $C_{P_{\nu}(K)}(H)$ conjugate to a cocharacter $\rho$ which commutes with $\lambda_{1}$. Then $P_{\rho} \cap P_{\nu}=P_{\tau} \cap P_{\nu}$ so $P_{\rho}$ is still opposite to $P_{\nu}$, and $H \leq L_{\rho}$. Now $\rho$ and $\nu$ both commute with $\lambda_{1}$, and so the images of these cocharacters are all contained in some maximal torus of $K$, call it $S$. Since $\lambda=n_{1} \lambda_{1}+n_{\nu} \nu$, the image of $\lambda$ also lies in $S$.

Now, again by the uniqueness of opposite R-parabolic subgroups among those containing a given maximal torus of $K$, it follows that we can scale $\rho$ so that $\rho=-n_{\nu} \nu$ and thus $-\lambda=-n_{1} \lambda_{1}-n_{\nu} \nu=-n_{1} \lambda_{1}+\rho$ as elements of $Y(S)$. This shows that $P_{-\lambda}=P_{-\lambda_{1}} \cap P_{\rho}$ is opposite to $P_{\lambda}$, lies in $\mathcal{P}_{K}^{\prime}$ and contains $H$, as required.

Proof of Theorem 1.2. The equivalence of the conditions (i) and (iii) follows immediately from [4, Prop. 3.17(ii)]. Now suppose (i) holds and let $P \in \mathcal{M}_{K}$ with $P \geq H$. By hypothesis, there exists an R-parabolic subgroup $Q \in \mathcal{P}_{K}$ which is opposite to $P$ and contains $H$, and then $Q \in \mathcal{M}_{K}$ by Lemma 2.7, so condition (ii) holds. Note that we have not yet used the hypothesis that $G$ is connected. 
Finally, suppose that (ii) holds, so that each $P \in \mathcal{M}_{K}$ which contains $H$ admits an opposite in $\mathcal{M}_{K}$ which contains $H$. Since $G$ is connected by hypothesis, we have $\mathcal{P}_{K}=\mathcal{P}_{K}^{\prime}$ by Lemma 2.2(ii). Then Theorem 1.4 tells us that each $P \in \mathcal{P}_{K}$ containing $H$ has an opposite in $\mathcal{P}_{K}$ containing $H$, hence condition (i) holds.

\section{The CASE $G=\mathrm{GL}(V)$}

We take this opportunity to note some interesting special cases of our results in the case $G=\mathrm{GL}(V)$. The first follows immediately from Lemma 2.2(i). Recall that $\mathcal{F}_{K}$ denotes the set of flags arising from parabolic subgroups corresponding to cocharacters of $K$, and $\mathcal{M F}_{K}$ denotes the minimal members of $\mathcal{F}_{K}$ (i.e. those whose stabilizers lie in $\mathcal{M}_{K}$ ).

Corollary 4.1. Let $G=\mathrm{GL}(V)$, let $K \leq G$ be a reductive subgroup, let $f$ be a flag in $\mathcal{F}_{K}$ and let $U$ be a subspace in $f$. Then there is a flag $f^{\prime}$ in $\mathcal{M F}_{K}$ such that $f^{\prime} \preceq f$ and $U$ appears in $f^{\prime}$.

Recall that $\mathcal{S}_{K}$ is the set of subspaces of $V$ which appear in flags from $\mathcal{F}_{K}$. The following is an immediate consequence of Corollary 4.1 .

Corollary 4.2. Let $K$ be a reductive subgroup of $\mathrm{GL}(V)$. Then the following are equivalent:

(i) $\mathcal{S}_{K}=\left\{U \subseteq V \mid(U \subseteq V) \in \mathcal{F}_{K}\right\}$.

(ii) $\mathcal{M F}_{K} \subseteq \mathcal{M} \mathcal{F}_{\mathrm{GL}(V)}$.

Next, we note that the implication (i) $\Rightarrow$ (ii) of Corollary 1.6 fails without the hypothesis on $\mathcal{M F}_{K}$, as the following example illustrates.

Example 4.3. Let $G=\mathrm{GL}_{4}(k)$ and let $K$ be the subgroup of diagonal matrices of the form $\operatorname{diag}\left(t, s, s^{-1}, t^{-1}\right)$ with $s, t \in k^{*}$. Let $e_{1}, \ldots, e_{4}$ be the standard basis of $k^{4}$ and $U=$ $\left\langle e_{1}, e_{2}, e_{3}\right\rangle$. Suppose that $H$ is the parabolic subgroup of $G$ corresponding to the flag $U \subseteq V$. Since the flags from $\mathcal{F}_{K}$ have subspaces of dimension $(2,4),(1,3,4)$ and $(1,2,3,4)$, the group $H$ is not contained in $P_{\lambda}$ for any $\lambda \in Y(K) \backslash\{1\}$. Hence trivially, $H$ is relatively $G$-cr with respect to $K$. Note that $U \in \mathcal{S}_{K}$ and $H$ stabilizes $U$. One checks that the complement to $U$ in the set $\mathcal{S}_{K}$ is $W=\left\langle e_{4}\right\rangle$. But $H$ does not stabilize $W$.

Remark 4.4. Let $G=\mathrm{GL}(V)$. If $\mathcal{M F}_{K} \nsubseteq \mathcal{M F}_{G}$ then there exists a subgroup $H$ of $G$ such that $H$ is relatively $G$-cr with respect to $K$ and $H$ stabilizes a subspace $U^{\prime} \in \mathcal{S}_{K}$ but does not stabilize any complement to $U^{\prime}$. To see this, note that since $\mathcal{S}_{K} \neq\left\{U \subseteq V \mid(U \subseteq V) \in \mathcal{F}_{K}\right\}$, by Corollary 4.2, there exists a $U^{\prime}$ in $\mathcal{S}_{K}$ such that $\left(U^{\prime} \subseteq V\right) \notin \mathcal{F}_{K}$. Set $H:=\operatorname{Stab}_{G}\left(U^{\prime} \subseteq V\right)$. Then $H$ is not contained in $P_{\lambda}$ for any $\lambda \in Y(K) \backslash\{1\}$. Trivially, $H$ is relatively $G$-cr with respect to $K$. Note that $H$ stabilizes $U^{\prime}$ in $\mathcal{S}_{K}$ but does not stabilize any complement to $U^{\prime}$, since $H$ is a maximal parabolic subgroup of $G$.

For a fixed subspace $U \subseteq V$, we show in the following lemma that $K=\operatorname{GL}(U)$ satisfies the condition in Corollary 4.2(i). A maximal torus of $G$ also satisfies the condition. So Corollary 1.6 applies in these instances, thanks to Corollary 4.2 .

Lemma 4.5. Let $G=\mathrm{GL}(V)$ and let $U \subseteq V$. Fix a complement $\widetilde{U}$ to $U$ in $V$. Let $K=\mathrm{GL}(U) \leq G$, embedded via the decomposition $V=U \oplus \widetilde{U}$. Then $\mathcal{S}_{K}=\{W \subseteq V \mid(W \subseteq$ $\left.V) \in \mathcal{F}_{K}\right\}$. 
Proof. Let $\left(W_{1} \subseteq \ldots \subseteq W_{m} \subseteq V\right)$ be in $\mathcal{F}_{K}$. One sees by inspection that for each $1 \leq i \leq m$ we have $W_{i} \subseteq U$ or $\widetilde{U} \subseteq W_{i}$. On the other hand, suppose that $W$ is a subspace contained in $U$. Then we can find a complement $W^{\prime}$ to $W$ containing $\widetilde{U}$ and the cocharacter which acts with weight 1 on $W$ and weight 0 on $W^{\prime}$ lies in $Y(K)$ and affords the flag $(W \subseteq V) \in \mathcal{F}_{K}$. Similarly, all flags $(W \subseteq V)$ with $\widetilde{U} \subseteq W$ are in $\mathcal{F}_{K}$. Hence

$$
\mathcal{F}_{K}=\left\{\left(W_{1} \subseteq \ldots \subseteq W_{m} \subseteq V\right) \in \mathcal{F}_{G} \mid W_{i} \subseteq U \text { or } \widetilde{U} \subseteq W_{i} \text { for } 1 \leq i \leq m \text {, for some } m\right\},
$$

and so

$$
\mathcal{S}_{K}=\left\{W^{\prime} \subseteq V \mid W^{\prime} \subseteq U \text { or } \widetilde{U} \subseteq W^{\prime}\right\}=\left\{W \subseteq V \mid(W \subseteq V) \in \mathcal{F}_{K}\right\},
$$

as claimed.

In view of (4.6) and Corollary 4.2, Corollary 1.6 and Lemma 4.5 imply [4, Prop. 5.1]. So Corollary 1.6 may be viewed as a generalization of the special case treated in [4, Prop. 5.1].

Corollary 1.7 considers situations when $K$ acts irreducibly on $V$. We close this section with a characterization of relative $\mathrm{GL}(V)$-complete reducibility in case $V$ decomposes as a direct sum of $K$-modules. We first note a consequence of Lemma 2.6 which characterizes relative $G$-complete reducibility when $G$ and $K$ admit compatible direct-product structures.

Corollary 4.7. For $i=1,2$, let $K_{i} \subseteq G_{i}$ be reductive groups, $G:=G_{1} \times G_{2}$ and $K:=$ $K_{1} \times K_{2}$. Let $H \subseteq G$ be a subgroup. Then $H$ is relatively $G$-completely reducible with respect to $K$ if and only if $H$ is relatively $G$-completely reducible with respect to $K_{i}$ for $i=1,2$.

Proof. Let $\lambda \in Y\left(K_{1}\right)$ such that $H \leq P_{\lambda}$. By the proof of [3, Lem. 2.12], the parabolic subgroups of $G$ arising from cocharacters of $K$ have the form $P_{\lambda_{1}} \times P_{\lambda_{2}}$ with $\lambda_{i} \in Y\left(K_{i}\right)$ for $i=1,2$, since $G=G_{1} \times G_{2}$ and $K=K_{1} \times K_{2}$. Hence $P_{\lambda}=P_{\lambda}\left(G_{1}\right) \times G_{2}$. Since $H$ is relatively $G$-completely reducible with respect to $K$, there exists a $u=\left(u_{1}, u_{2}\right) \in R_{u}\left(P_{\lambda}(K)\right)$ such that $H \leq L_{u \cdot \lambda}=L_{u_{1} \cdot \lambda}\left(G_{1}\right) \times G_{2}$, by Lemma 2.6(ii). Therefore, $u_{1} \in R_{u}\left(P_{\lambda}\left(K_{1}\right)\right)$. It follows that $H$ is relatively $G$-cr with respect to $K_{1}$, by Lemma 2.6(ii). The proof for $K_{2}$ is analogous.

For the reverse implication let $\lambda=\left(\lambda_{1}, \lambda_{2}\right) \in Y(K)=Y\left(K_{1}\right) \times Y\left(K_{2}\right)$ such that $H \leq$ $P_{\lambda_{1}} \times P_{\lambda_{2}}$. Since $H$ is relatively $G$-completely reducible with respect to $K_{i}$ for $i=1,2$, there exits a $u_{i} \in R_{u}\left(P_{\lambda_{i}}\left(K_{i}\right)\right)$ such that $H \leq L_{u_{1} \cdot \lambda_{1}}\left(G_{1}\right) \times G_{2}$ resp. $H \leq G_{1} \times L_{u_{2} \cdot \lambda_{2}}\left(G_{2}\right)$. Therefore, we obtain

$$
H \leq\left(L_{u_{1} \cdot \lambda_{1}}\left(G_{1}\right) \times G_{2}\right) \cap\left(G_{1} \times L_{u_{2} \cdot \lambda_{2}}\left(G_{2}\right)\right)=L_{u \cdot \lambda}
$$

for $u=\left(u_{1}, u_{2}\right) \in R_{u}\left(P_{\lambda}(K)\right)$. Once again, by Lemma 2.6(ii), $H$ is relatively $G$-cr with respect to $K$.

The following result is now immediate from Corollary 4.7 and [4, Cor. 3.6].

Corollary 4.8. Let $G=\mathrm{GL}(V)$ and suppose that both $H$ and $K$ preserve a direct-sum decomposition $V=\bigoplus_{i=1}^{n} V_{i}$. Suppose also that $K=K_{1} \times \cdots \times K_{n}$ where $K_{i} \leq \mathrm{GL}\left(V_{i}\right)$ for each $i$. Then $H$ is relatively $G$-completely reducible with respect to $K$ if and only if $H$ is relatively $G$-completely reducible with respect to $K_{i}$ for all $i$.

Both implications in Corollary 4.8 fail in general, as illustrated by our next example. 
Example 4.9. Let $G=\mathrm{GL}\left(k^{4}\right), K=\left\{\operatorname{diag}\left(t, s, s^{-1}, t^{-1}\right) \mid t, s \in k^{*}\right\}$, and $\left\{e_{1}, e_{2}, e_{3}, e_{4}\right\}$ is the canonical basis for $k^{4}$. Set $V_{1}=\left\langle e_{1}, e_{2}\right\rangle$ and $V_{2}=\left\langle e_{3}, e_{4}\right\rangle$. Let $K_{i}$ be the image of the projection from $K$ to $\mathrm{GL}\left(V_{i}\right)$ for $i=1,2$.

Let $H$ be the stabilizer of $U:=\left\langle e_{2}, e_{4}\right\rangle$ in $G$ and note that $\left(U \subseteq k^{4}\right)$ belongs to $\mathcal{F}_{K}$. Thus $H$ is a maximal parabolic subgroup of $G$ and corresponds to a cocharacter of $K$, and as such it is not relatively $G$-cr with respect to $K$. However, $H$ does not correspond to a cocharacter of $K_{i}$, and by maximality $H$ is not contained in any parabolic subgroup of $G$ correspond to a cocharacter of $K_{i}(i=1,2)$. Hence $H$ is relatively $G$-irreducible with respect to $K_{i}$ so it is relatively $G$-cr with respect to $K_{i}$, for $i=1,2$.

Now let $\widetilde{H}$ be the stabilizer of $\widetilde{U}:=\left\langle e_{1}\right\rangle$ in $G$. Note that $\widetilde{H}$ is a maximal parabolic subgroup of $G$ and corresponds to a cocharacter of $K_{1}$, thus it is not relatively $G$-cr with respect to $K_{1}$. However, since $\widetilde{H}$ does not correspond to a cocharacter of $K$, it is relatively $G$-irreducible with respect to $K$, in particular it is relatively $G$-cr with respect to $K$.

\section{Rationality Questions}

In this section $k$ denotes an arbitrary field, $G$ is a reductive $k$-defined group and $K$ is a reductive $k$-defined subgroup of $G$. For a $k$-defined closed subgroup $M$ of $G$, write $Y_{k}(M)$ for the $k$-defined cocharacters of $M$, and let $M(k)$ denote the group of $k$-points of $M$. First, we recall the definition of relative $G$-complete reducibility over $k$ from [4, Def. 4.1], and also define the analogue of relative $G$-irreducibility over $k$.

Definition 5.1. Let $H$ be a subgroup of $G$. We say that $H$ is relatively $G$-completely reducible over $k$ with respect to $K$ if for every $\lambda \in Y(K)$ such that $P_{\lambda}$ is $k$-defined and $H$ is contained in $P_{\lambda}$, there exists $\mu \in Y(K)$ such that $P_{\lambda}=P_{\mu}, H$ is contained in $L_{\mu}$ and $L_{\mu}$ is $k$-defined. We also say that $H$ is relatively $G$-irreducible over $k$ with respect to $K$ if $H$ is not contained in any $k$-defined parabolic subgroup $P_{\lambda}$ with $\lambda \in Y(K)$.

Remark 5.2. By [4, Lem. 4.8], a subgroup is relatively $G$-cr over $k$ with respect to $K$ if and only if for every $\lambda \in Y_{k}(K)$ such that $H \leq P_{\lambda}$, there exists $\mu \in Y_{k}(K)$ such that $P_{\lambda}=P_{\mu}$ and $H \leq L_{\mu}$. By identical arguments to those in the proof of [4, Lem. 4.8], a subgroup is relatively $G$-irreducible over $k$ with respect to $K$ if and only if it is not contained in any R-parabolic subgroup $P_{\lambda}$ with $\lambda \in Y_{k}(K)$.

Analogous to Theorem 1.1, we have a geometric characterization of relative $G$-complete reducibility over $k$. We recall some definitions [2, Def. 1.1], [5, Def. 5.4].

Definition 5.3. Let $G$ be reductive and $k$-defined.

(i) Let $G$ act $k$-morphically on an affine $k$-variety $X$, and let $x \in X$. The orbit $G(k) \cdot x$ is called cocharacter-closed over $k$ if for all $\lambda \in Y_{k}(G)$ such that $\lim _{a \rightarrow 0} \lambda(a) \cdot x$ exists, then this limit lies in the $G(k)$-orbit $G(k) \cdot x$.

(ii) A generic tuple for a subgroup $H$ of $G$ is an $n$-tuple $\left(h_{1}, \ldots, h_{n}\right) \in G^{n}$ such that $H$ and $\left\{h_{1}, \ldots, h_{n}\right\}$ generate the same associative subalgebra of $\operatorname{Mat}_{m \times m}(\bar{k})$, for some embedding $G \rightarrow \mathrm{GL}_{m}(\bar{k})$. 
Note that a generic tuple for $H$ always exists since $\operatorname{Mat}_{m \times m}(\bar{k})$ is a finite-dimensional $\bar{k}$-algebra. The following summarizes part of [4, Thm. 4.12(iii)].

Theorem 5.4. Let $K$ be a reductive subgroup of $G$, let $H \leq G$ and let $\mathbf{h} \in G^{n}$ be a generic tuple for $H$. Then $H$ is relatively $G$-completely reducible over $k$ with respect to $K$ if and only if $K(k) \cdot \mathbf{h}$ is cocharacter-closed over $k$.

We now generalize Theorems 1.2 and 1.4 to the rational setting. First, we need a rational analogue of [4, Prop. 3.17].

Proposition 5.5. Let $K$ be a $k$-defined reductive subgroup of $G$, and let $H \leq G$.

(i) Suppose that $H \leq L:=L_{\lambda}$ where $\lambda \in Y_{k}(K)$. Then $H$ is relatively $G$-completely reducible over $k$ with respect to $K$ if and only if $H$ is relatively L-completely reducible over $k$ with respect to $K \cap L$.

(ii) If $L$ is minimal among subgroups of the form $L_{\lambda}$ with $\lambda \in Y_{k}(K)$ containing $H$, then $H$ is relatively $G$-completely reducible over $k$ with respect to $K$ if and only if $H$ is relatively $L$-irreducible over $k$ with respect to $K \cap L$.

Proof. Noting that the image of $\lambda$ is a $k$-split torus, part (i) follows directly from [2, Thm. 5.4(ii)] applied to the $K$-orbit of $\mathbf{h} \in G^{n}$, where $\mathbf{h} \in G^{n}$ is a generic tuple for $H$. For part (ii), we have shown that $H$ is relatively $L$-cr over $k$ with respect to $K \cap L$. But also, from the minimality of $L$, it follows that any $k$-defined cocharacter of $K \cap L$ centralized by $H$ is central in $L$, hence $H$ is relatively $L$-irreducible over $k$ with respect to $K \cap L$.

We are now in a position to prove the rational counterparts of Theorems 1.2 and 1.4. Define the following analogues of the sets $\mathcal{P}_{K}, \mathcal{M}_{K}$ and $\mathcal{P}_{K}^{\prime}$ :

$$
\begin{aligned}
\mathcal{P}_{K, k} & =\left\{P_{\mu} \mid \mu \in Y_{k}(K)\right\}, \\
\mathcal{M}_{K, k} & =\left\{\text { inclusion-maximal members of } \mathcal{P}_{K, k}\right\}, \\
\mathcal{P}_{K, k}^{\prime} & =\left\{P \in \mathcal{P}_{K, k} \mid P=\bigcap\left\{Q \in \mathcal{M}_{K, k} \mid P \leq Q\right\}\right\} .
\end{aligned}
$$

With these definitions, the obvious analogue of Lemma 2.2 holds for connected $G$. In the proof, one needs to work with the relative root system of $G$ with respect to a maximal $k$ split torus [6, V.21], [9, $§ 15,16]$, but otherwise the argument goes through mutatis mutandis. The conclusion of Lemma 2.4 also holds when considering only $k$-defined cocharacters. Since $\mathcal{M}_{K, k}=\mathcal{M}_{K} \cap \mathcal{P}_{K, k}$ and $\mathcal{P}_{K, k}^{\prime}=\mathcal{P}_{K}^{\prime} \cap \mathcal{P}_{K, k}$, Lemma 2.7 immediately implies its own rational analogue. Finally, [4, Lem. 4.6] shows that each time we use Lemma 2.6 to conjugate a subgroup or cocharacter, the conjugating element can be taken to be a $k$-point.

The following are now the rational versions of Theorem 1.2 and 1.4.

Theorem 5.6. Let $K \leq G$ be reductive $k$-defined algebraic groups with $G$ connected, and let $H$ be a subgroup of $G$. Then the following are equivalent.

(i) $H$ is relatively $G$-completely reducible over $k$ with respect to $K$.

(ii) Every member of $\mathcal{M}_{K, k}$ containing $H$ has an opposite in $\mathcal{M}_{K, k}$ containing $H$.

(iii) There is an $R$-Levi subgroup $L_{\mu}$ with $\mu \in Y_{k}(K)$, such that $H \leq L_{\mu}$ and $H$ is relatively $L_{\mu}$-irreducible over $k$ with respect to $K \cap L_{\mu}$. 
Theorem 5.7. Let $K \leq G$ be reductive $k$-defined algebraic groups, and let $H$ be a subgroup of $G$. Then each member of $\mathcal{M}_{K, k}$ containing $H$ has an opposite in $\mathcal{M}_{K, k}$ containing $H$ if and only if each member of $\mathcal{P}_{K, k}^{\prime}$ containing $H$ has an opposite in $\mathcal{P}_{K, k}^{\prime}$ containing $H$.

Remark 5.8. By Remark 5.2, we could equally well take $\mathcal{P}_{K, k}$ to be the set of $k$-defined R-parabolic subgroups of the form $P_{\lambda}$ with $\lambda \in Y(K)$ and $\mathcal{M}_{K, k}$ to be the set of maximal members of this $\mathcal{P}_{K, k}$. However, working with R-parabolic and R-Levi subgroups corresponding to elements of $Y_{k}(K)$ allows our proofs to be more naturally generalized.

Proof of Theorems 5.7 and 5.6. The rational analogue of Lemma 2.7 gives one direction of Theorem 5.7. For the reverse direction, the rational analogues of Lemmas 2.4 and 2.6 are almost sufficient for the proof of Theorem 1.4 to go through mutatis mutandis. The only subtle point is when conjugating a cocharacter by an element of ' $C_{P_{\nu}(K)}(H)$ '; we need the conjugating element to lie in $C_{P_{\nu}(K)}(H)(k)$. That we can guarantee this follows from the conjugacy of maximal $k$-split tori in [2, Lem. 2.12].

Thus Theorem 5.7 holds. In Theorem 5.6, the equivalence of conditions (i) and (iii) follows from Proposition 5.5. The implication (i) $\Rightarrow$ (ii) follows from the rational analogue of Lemma 2.7. As in the algebraically closed case, these implications do not use the fact that $G$ is connected. Finally, if $G$ is connected then the rational version of Lemma 2.2 tells us that $\mathcal{P}_{K, k}=\mathcal{P}_{K, k}^{\prime}$, and so the implication (ii) $\Rightarrow$ (i) follows from Theorem 5.7.

\section{Appendix A. Extended example: $G_{2}$ IN $_{\mathrm{GL}_{7}}$}

We close by considering a non-trivial example of Corollary 1.5 (and hence Theorem 1.2) when $V$ is a faithful irreducible module for a simple algebraic group of exceptional type.

Fix the algebraically closed field $k$ and let $K$ be a simple algebraic group of type $G_{2}$ over $k$. Then $K$ can be realized as the group of invertible linear transformations of a 7 -dimensional $k$-vector space $V$ which preserve an alternating trilinear form and associated bilinear form (or quadratic form if $k$ has characteristic 2) [1, §3].

By [1, Thms. 1,3], the R-parabolic subgroups $P_{\lambda}(K)$ of $K$, with $\lambda \in Y(K)$, are precisely the stabilizers in $K$ of doubly singular subspaces of $V$ of dimension 1 or 2, where doubly singular means singular with respect to both the trilinear form and the bilinear (or quadratic) form. By [1, Thm. 2], $K$ is transitive on such subspaces of each dimension.

Now let $G=\mathrm{GL}(V)$. Elementary calculations with high weights show that a given maximal torus of $K$ is $G$-conjugate to the subtorus

$$
S=\left\{\operatorname{diag}\left(s, t, s t^{-1}, 1, s^{-1} t, t^{-1}, s^{-1}\right) \mid s, t \in k^{*}\right\}
$$

of $G$, with respect to an appropriate basis of $V$. It then follows quickly that flags of subspaces of $V$ corresponding to a cocharacter $\lambda \in Y(S)$ involve intermediate subspaces of dimensions $\{2,5\},\{1,3,4,6\}$ and $\{1,2,3,4,5,6\}$. In the first two cases, $P_{\lambda}(K)$ is a maximal parabolic subgroup of $K$ and $P_{\lambda} \in \mathcal{M}_{K}$. In the third case $P_{\lambda}(K)$ and $P_{\lambda}$ are respectively Borel subgroups of $K$ and of $G$. The flags of type $\{2,5\}$ consist of doubly singular subspaces of $V$ and their annihilators, and $K$ is transitive on these. By [1, $\S \S 4.2]$ the flags of type $\{1,3,4,6\}$ can be described as those of the form $U \subseteq \Delta(U) \subseteq \Delta(U)^{\perp} \subseteq U^{\perp}$, where $U=\langle x\rangle$ is 1-dimensional and doubly singular and the 3-dimensional subspace $\Delta(U)$ is defined as the 
radical of the bilinear form $(u, v)=f(x, u, v)$ with $f$ the $K$-stable trilinear form. Since $K$ is transitive on the subspaces $U$ it is also transitive on these flags.

Explicitly writing out condition (ii) of Theorem 1.2 yields the following criteria for relative complete reducibility in this scenario.

Theorem A.1. With the above notation, a subgroup $H$ of $G=\mathrm{GL}(V)$ is relatively $G$ completely reducible with respect to the subgroup $K$ of type $G_{2}$ if and only if the following two conditions hold.

(i) If $H$ stabilizes a flag $U \subseteq U^{\perp}$ where $U$ is 2-dimensional and doubly singular, then $H$ also stabilizes a flag $W \subseteq W^{\perp}$ where $W$ is 2-dimensional and doubly singular, and $V=U \oplus W^{\perp}=W \oplus U^{\perp}$.

(ii) If $H$ stabilizes a flag $U \subseteq \Delta(U) \subseteq \Delta(U)^{\perp} \subseteq U^{\perp}$ where $U$ is 1-dimensional and doubly singular, then $H$ stabilizes another such flag $W \subseteq \Delta(W) \subseteq \Delta(W)^{\perp} \subseteq W^{\perp}$ with

$$
V=U \oplus W^{\perp}=W \oplus U^{\perp}=\Delta(U) \oplus \Delta(W)^{\perp}=\Delta(W) \oplus \Delta(U)^{\perp} .
$$

For example, let $U \subseteq U^{\perp}$ be a flag as in (i) and let $H$ be any Levi subgroup of the corresponding parabolic subgroup of $\mathrm{GL}(V)$. Then $H \cong \mathrm{GL}_{2} \times \mathrm{GL}_{3} \times \mathrm{GL}_{2}$ and $H$ does not stabilize a flag of subspaces of the form in (ii). Thus $H$ is relatively $G$-cr with respect to $G_{2}$ if and only if the complementary flag stabilized by $H$ also has the form described in (i).

Remark A.2. The construction of Chevalley groups of type $G_{2}$ given in [1] holds for arbitrary fields $k$, and produces the same description of the maximal parabolic subgroups thereof. Thus for split groups of type $G_{2}$ over any field $k$, the analogue of Theorem A.1 holds, characterising relative complete reducibility over $k$. We thank the anonymous referee for pointing this out.

\section{REFERENCES}

[1] M. Aschbacher, Chevalley groups of type $G_{2}$ as the group of a trilinear form, J. Alg. 109 (1987), no. 1, pp. 193-259.

[2] M. Bate, S. Herpel, B. Martin, G. Röhrle, Cocharacter-closure and the rational Hilbert-Mumford Theorem, Math. Z. 287 (2017), no. 1-2, 39-72.

[3] M. Bate, B. Martin, G. Röhrle, A geometric approach to complete reducibility, Invent. Math. 161, no. 1 (2005), 177-218.

[4] M. Bate, B. Martin, G. Röhrle, R. Tange, Complete reducibility and conjugacy classes of tuples in algebraic groups and Lie algebras, Math. Z. 269 (2011), 809-832.

[5] _ Closed orbits and uniform S-instability in geometric invariant theory, Trans. Amer. Math. Soc., 365 no. 7 (2012), 3643-3673.

[6] A. Borel, Linear algebraic groups, Graduate Texts in Mathematics, 126, Springer-Verlag 1991.

[7] R.W. Richardson, Conjugacy classes of $n$-tuples in Lie algebras and algebraic groups, Duke Math. J. 57, (1988), no. 1, 1-35.

[8] J-P. Serre, Complète réductibilité, Séminaire Bourbaki, 56ème année, 2003-2004, nº 932.

[9] T.A. Springer, Linear algebraic groups, Second edition. Progress in Mathematics, 9. Birkhäuser Boston, Inc., Boston, MA, 1998.

Christopher Attenborough: Department of Mathematics, University of York, York YO10 5DD, United Kingdom

E-mail address: cea522@york.ac.uk 
Michael Bate: Department of Mathematics, University of York, York YO10 5DD, United KINGDOM

E-mail address: michael.bate@york.ac.uk

Maike Gruchot: Fakultät für Mathematik, Ruhr-Universität Bochum, D-44780 Bochum, Germany

E-mail address: maike.gruchot@rub.de

Current address: Lehrstuhl D für Mathematik, RWTH Aachen University, D-52062 Aachen, Germany

E-mail address: maike.gruchot@rwth-aachen.de

Alastair Litterick: Fakultät für Mathematik, Ruhr-Universität Bochum, D-44780 Bochum, Germany, and Fakultät für Mathematik, Universität Bielefeld, D-33501 Bielefeld, GerMANY

E-mail address: alastair.litterick@rub.de

Current address: Department of Mathematical Sciences, University of Essex, Wivenhoe Park, Colchester, Essex CO4 3SQ, United Kingdom

E-mail address: ajlitterick@essex.ac.uk

Gerhard Röhrle: Fakultät für Mathematik, Ruhr-Universität Bochum, D-44780 Bochum, Germany

E-mail address: gerhard.roehrle@rub.de 